\title{
Metronidazole Induced Encephalopathy with Peripheral Polyneuropathy in Patient with Spinal Cord Injury
}

\author{
Gi Hoon Hwang', Young-Joo Sim¹, Ho Joong Jeong ${ }^{1}$, \\ Ghi Chan Kim ${ }^{1}$, Bae Wook $\mathrm{Sin}^{1}$, Ju Ho Jung ${ }^{2}$ \\ Departments of Physical Medicine and Rehabilitation ${ }^{1}$, Neurosurgery ${ }^{2}$, Kosin University College of Medicine, Busan, Korea
}

Metronidazole may produce a number of neurologic side effects including peripheral neuropathy, seizure, encephalopathy. We experienced neurological side effects of metronidazole. The 32-year-old female patient with spinal cord injury was diagnosed as encephalophathy and peripheral polyneuropathy resulting from complication of metronidazole. It was difficult to diagnose at first glance using clinical findings because of paraplegia due to spinal cord injury. But through magnetic resonance imaging with diffusion weighted imaging and electrophysiologic study, the patient showed to have characteristic abnormalities that of a person suffering from metronidazole-induced encephalopathy and peripheral polyneuropathy. Whether the symptoms were caused by a peripheral nerve lesion or MIE, the patient's paraplegia prevented to appear other symptoms, such as ataxic gait and seizure, from manifesting. In such case as this, an active differentiated diagnosis is crucial.

Key Words: Metronidazole $\cdot$ Encephalopathy $\cdot$ Spinal cord injury

\section{INTRODUCTION}

Metronidazole is a commonly used antimicrobial agent used for the treatment of protozoal and bacterial infection. This drug is in its inactive state when administered to a patient, allowing selective targeting. Once in the system the drug is taken up by the target and metabolized into its active form, inhibiting its normal function. The most common side effects of metronidazole include nausea, headaches and the metallic taste in the mouth. In some cases, high doses and/or prolong use may produce several neurologic side effects including peripheral neuropathy, cerebellopathy, encephalopathy and seizure $^{2,3,5,7)}$.

Recently, we encountered a case of metronidazole-induced encephalopathy (MIE) with peripheral polyneuropathy (PPN) in patient with spinal cord injury (SCI). MIE with PPN is a very rare occurrence let alone a MIE on a patient with spinal cord injury, which has not been previously documented. Whether the encephalopathy and PPN was induced by metronida-

- Received: January 18, 2012 - Revised: March 26, 2012

- Accepted: March 28, 2012

Corresponding Author: Young-Joo Sim, MD

Department of Physical Medicine and Rehabilitation, Kosin University

College of Medicine, 34, Amnam-dong, Seo-gu, Busan 602-702, Korea

Tel: +82-51-990-6156, Fax: +82-51-241-2019

E-mail: oggum@hanmail.net zole or some other factor in patient with spinal cord injury, diagnosis at first glance proved to be very difficult. This was because the sure signs (ataxia, gait difficulty, tingling sensation in lower extremity and so on) of the drug's side effects were not present due to the paraplegia. However, observing this patient carefully, we have noticed something that might serve as detection for MIE with PPN despite paraplegia.

\section{CASE REPORT}

A 32-year-old woman injured in a skiing accident a month before her visit, was transferred to our institute from another hospital as a result of her paraplegia. Initial workshop at the other hospital showed bursting fractures of T6, T7, T8 vertebral bodies, hemothorax and multiple rib fractures (Fig. 1). Neurologic examination revealed a motor and sensory impairment of grade A at the T6 level, according to the American Spinal Injury Association (ASIA) scale. She was examined for vaginal discharge at the previous hospital and was diagnosed with erosion and acute inflammation of the cervix. She was then treated with $2 \mathrm{~g}$ of metronidazole daily for fifty days because of the vaginal discharge.

One month after the first administration of metronidazole, she complained of a mild tingling sensation on both forearms and all fingers as stocking glove type. The patient denied other symptoms including neck pain and hand weakness. However 
laboratory findings from blood and urine samples did not show any specific abnormalities $(\mathrm{Hb}: 12.4 \mathrm{~g} / \mathrm{dL}$, Protein: 5.7 gm/dL, Albumin: $3.5 \mathrm{~g} / \mathrm{dl}$, GOT: 38 IU/L, GPT: $59 \mathrm{IU} / \mathrm{L}$, Na: $142 \mathrm{meq} / \mathrm{L}, \mathrm{K}: 4.3 \mathrm{meq} / \mathrm{L}$, Ca: $9.4 \mathrm{mg} / \mathrm{dL}, \mathrm{P}: 4.9 \mathrm{mg} / \mathrm{dL}$ ). We examined Tinnel and Phalen sign and proceeded to do an electrophysiological study in the upper extremities as well as a cervical spine MRI, checking for Carpal Tunnel Syndrome (CTS), syringomyelia, and other complications that might have risen in the patient with spinal cord injury. However the tests revealed normal finding.

Forty five days after the first treatment of metronidazole she developed mild nausea and vomiting with dizziness. We performed esophagogastroduodenoscopy to check for gastric ulcer, but observed only atrophic gastritis on a pyloric antrum. Fifty days after the first medication of metronidazole, we observed dysarthria and weakness of both hands. But, we did not

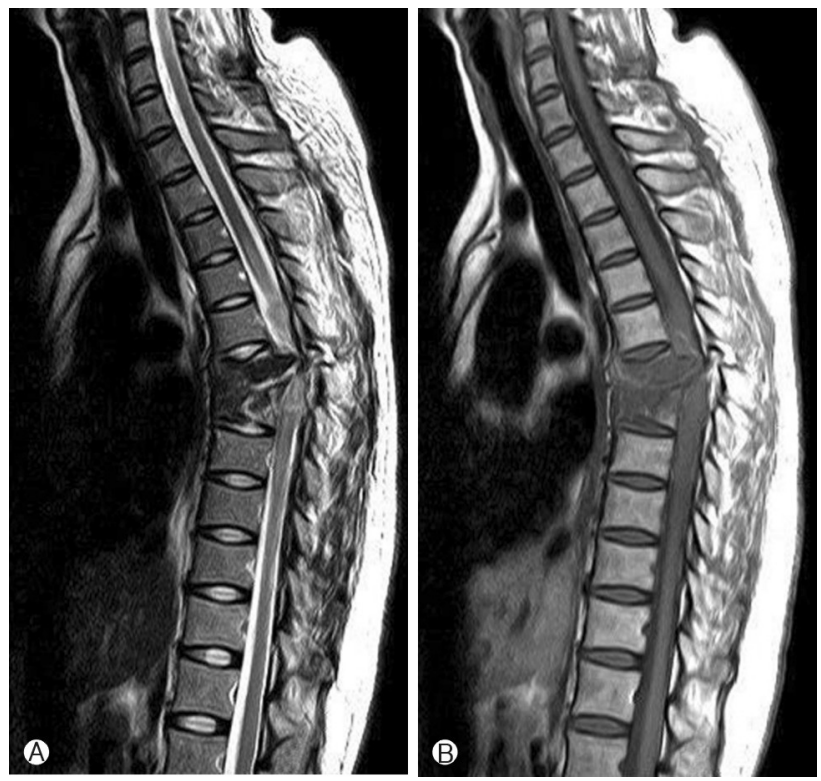

Fig. 1. T2-weighted image (A) and $\mathrm{T} 1$-weighted image $(\mathrm{B})$ reveal bursting fractures in the $\mathrm{T} 6$ and $\mathrm{T} 7$ vertebral bodies. find pathologic nystagmus and diadochokinesia beside dysmetria of hands. Because of paraplegia, we could not examine whether ataxia exist, or not. Laboratory findings from blood showed no abnormalities $(\mathrm{Hb}: 12.9 \mathrm{~g} / \mathrm{dL}$, WBC count: 7,200 uL ESR: $14 \mathrm{~mm} / \mathrm{hr}$, Protein: $5.8 \mathrm{gm} / \mathrm{dL}$, Albumin: $3.8 \mathrm{~g} / \mathrm{dl}$, GOT: 22 IU/L, GPT: $18 \mathrm{IU} / \mathrm{L}, \mathrm{Na}: 138 \mathrm{meq} / \mathrm{L}, \mathrm{K}: 3.9 \mathrm{meq} / \mathrm{L}$, Ca: $9.1 \mathrm{mg} / \mathrm{dL}$, P: $4.7 \mathrm{mg} / \mathrm{dL}$, Vit. B12: $611 \mathrm{pg} / \mathrm{mL}[200-950]$, Folate: $4.34 \mathrm{ng} / \mathrm{mL}[3-17])$. The brain magnetic resonanace image (MRI) with diffusion-weighted image (DWI) was performed to rule out brain lesions. We also performed electrophysiologe examinations of upper and lower limbs to find signs of peripheral polyneuropathy, simultaneously. We observed high intensities of superior olivary nuclei, dentate nuclei of cerebellum and splenium of corpus callosum on MRI (Figs. 2 and 3). In addition, the findings of electrophysiological examination revealed that amplitudes (base to peak amplitude) were below normal or absent on sensory nerves of more than 3 limbs, on the other hand, onset latency and velocity of those were normal findings. Also latency, amplitude and conduction velocity of motor nerves were normal, but, conduction velocity of peroneal and tibial nerve were lower limit normal. Because of above results, we considered PPN as axonal loss sensory type in this case (Table 1 and 2).

Before we could suspect that metronidazole was behind the new symptoms, we first had to rule out Wernicke Encephalopathy (WE) because of brain MRI images. MIE and WE have similar histologic findings6; therefore it was necessary to differentiate the two. WE have a triad of symptoms including confusion, ophthalmoplegia and gait ataxia while MIE has dysarthria and gait ataxia. We examined the level of thiamine hydrochloride because its deficiency could result in WE. Showing no lack of thiamine hydrochloride, we correlated the MR imaging with the clinical symptoms and diagnosed her as having MIE with PPN and stopped the medication of metronidazole.

Two days later, dysarthria and nausea improved slowly but a little tingling sensation and weakness of both hands persisted for more than three months. Three month later, we rechecked
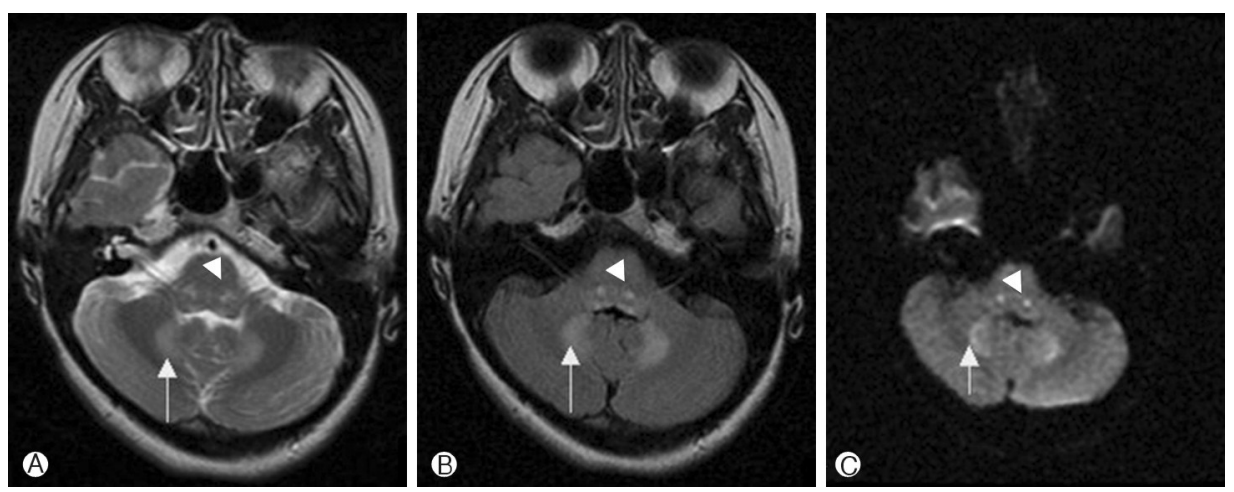

Fig. 2. T2-weighted (TR/TE $=4000$ / 115) image (A), FLAIR (TR/TE = $8802 / 161)$ image (B) and DW (TR/ $\mathrm{TE}=10000 / 122.2)$ image (C) reveal bilateral, symmetric, hyperintense lesions in the superior olivary nuclei of the dorsal pons (arrow heads) and in the dentate nuclei of the cerebellum (arrows). 

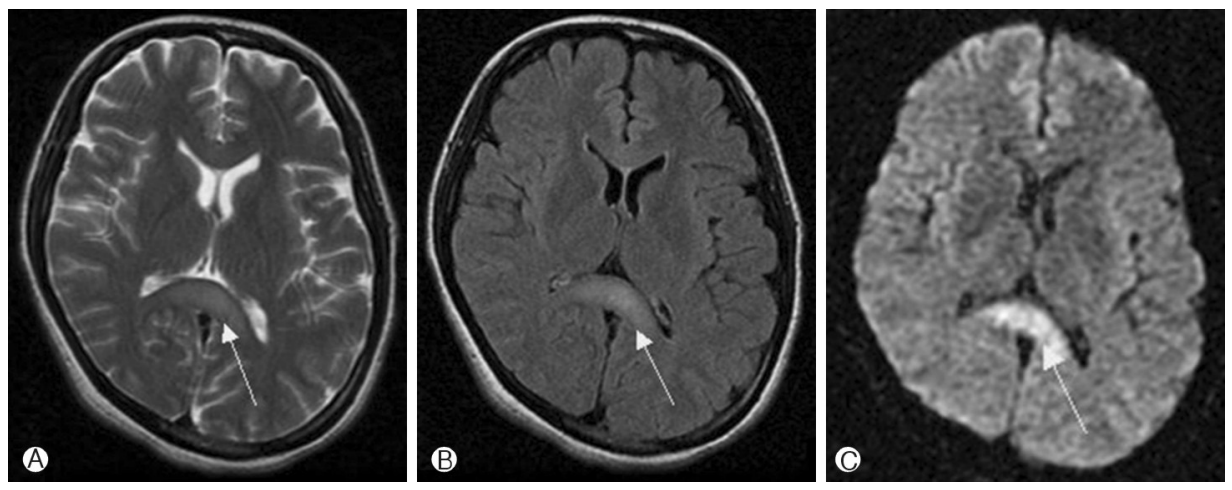

Fig. 3. T2-weighted (TR/TE $=4000 /$ 115) image (A), FLAIR (TR/TE= $8802 / 161)$ image (B) and DW (TR/ $\mathrm{TE}=10000 / 122.2)$ image (C) demonstrate a focal segmental lesion in the splenium of the corpus callosum (arrows).

Table 1. Motor and sensory nerve conduction study

\begin{tabular}{|c|c|c|c|c|}
\hline Nerve & Onset Latency (ms) & Peak Latency (ms) & Base to Peak Amplitude $(\mu \mathrm{V})$ & Conduction Velocity $(\mathrm{m} / \mathrm{s})$ \\
\hline \multicolumn{5}{|l|}{ Motor nerves } \\
\hline Rt. Median & $2.9(\leq 3.6)$ & & $8,200(\geq 5,000)$ & $49(\geq 50)$ \\
\hline Rt. Ulnar & $1.9(\leq 4.2)$ & & $10,000(\geq 5,000)$ & $53(\geq 50)$ \\
\hline Rt. Radial & $1.4(\leq 2.0)$ & & $6,700(\geq 5,000)$ & $60(\geq 50)$ \\
\hline Lt. Median & $2.5(\leq 3.6)$ & & $9,300(\geq 5,000)$ & $48(\geq 50)$ \\
\hline Lt. Ulnar & $1.5(\leq 4.2)$ & & $7,800(\geq 5,000)$ & $50(\geq 50)$ \\
\hline Lt. Radial & $1.2(\leq 2.0)$ & & $6,100(\geq 5,000)$ & $51(\geq 50)$ \\
\hline Rt. Peroneal & $3.4(\leq 6.0)$ & & $9,000(\geq 2,500)$ & $34(\geq 40)$ \\
\hline Rt. Tibial & $3.2(\leq 6.0)$ & & $2,200(\geq 5,000)$ & $37(\geq 40)$ \\
\hline Lt. Peroneal & $4.0(\leq 6.0)$ & & $8,300(\geq 2,500)$ & $39(\geq 40)$ \\
\hline Lt. Tibial & $2.4(\leq 6.0)$ & & $2,600(\geq 5,000)$ & $38(\geq 40)$ \\
\hline \multicolumn{5}{|l|}{ Sensory nerves } \\
\hline Rt. Median & $2.1(\leq 3.6)$ & 2.8 & $10(\geq 10)$ & $62(\geq 45)$ \\
\hline Rt. Ulnar & $1.8(\leq 3.4)$ & 2.5 & $6(\geq 10)$ & $58(\geq 45)$ \\
\hline Rt. Radial & $2.0(\leq 2.0)$ & 2.5 & $8(\geq 10)$ & $60(\geq 40)$ \\
\hline Lt. Median & $2.3(\leq 3.6)$ & 2.9 & $8(\geq 10)$ & $61(\geq 45)$ \\
\hline Lt. Ulnar & $1.8(\leq 3.4)$ & 2.6 & $8(\geq 10)$ & $62(\geq 45)$ \\
\hline Lt. Radial & $1.7(\leq 2.0)$ & 2.4 & $7(\geq 10)$ & $56(\geq 40)$ \\
\hline Rt. Sural & Absent $(\leq 3.5)$ & Absent & Absent $(\geq 10)$ & $-(\geq 40)$ \\
\hline Lt. Sural & Absent $(\leq 3.5)$ & Absent & Absent $(\geq 10)$ & $-(\geq 40)$ \\
\hline Rt. Superficial peroneal & Absent $(\leq 3.5)$ & Absent & Absent $(\geq 10)$ & $-(\geq 40)$ \\
\hline Lt. Superficial peroneal & Absent $(\leq 3.5)$ & Absent & Absent $(\geq 10)$ & $-(\geq 40)$ \\
\hline
\end{tabular}

The numeric values in parentheses refer to normal laboratory values. Rt., right; Lt., left.

the electrophysiological condition in the upper and lower extremity, and it did not revealed-some recovery.

\section{DISCUSSION}

Neurotoxicity is one of the more serious adverse effects of metronidazole therapy, but it is very rare. The types of neurological side effects ranges from frequent reports of reversible cerebellopathy and peripheral neuropathy, to other uncommon, but documented, neurotoxicities such as encephalopathy, seizure and mental confusion.

Ahmed et al. described the imaging findings of metronida- zole toxicity in 1995 in a 45-year-old woman who developed nausea, vomiting, dysarthria and confusion after consuming $35 \mathrm{~g}$ of metronidazole over a 30-day course of therapy ${ }^{1)}$. Horlen et al. reported imaging findings of presumed metronidazole toxicity in a 35 -year-old male patient with liver cirrhosis who had consumed greater than $60 \mathrm{~g}$ metronidazole over a 55-day period and developed clinical symptoms consistent with metronidazole toxicity in $2000^{5}$. Heaney et al. reported imaging findings of a 74-year-old male patient with progressive bilateral lower extremity weakness and dysarthria in $2003^{4}$. The patient had consumed a total of more than $75 \mathrm{~g}$ metronidazole over 8-weeks period.

In each of these cases, including ours, there was a symmetri- 
Table 2. Follow up motor and sensory nerve conduction study

\begin{tabular}{|c|c|c|c|c|}
\hline Nerve & Onset Latency (ms) & Peak Latency (ms) & Base to Peak Amplitude $(\mu \mathrm{V})$ & Conduction Velocity $(\mathrm{m} / \mathrm{s})$ \\
\hline \multicolumn{5}{|l|}{ Motor nerves } \\
\hline Rt. Median & $2.3(\leq 3.6)$ & & $10,000(\geq 5,000)$ & $53(\geq 50)$ \\
\hline Rt. Ulnar & $1.7(\leq 4.2)$ & & $10,100(\geq 5,000)$ & $56(\geq 50)$ \\
\hline Rt. Radial & $1.3(\leq 2.0)$ & & $5,600(\geq 5,000)$ & $60(\geq 50)$ \\
\hline Lt. Median & $2.6(\leq 3.6)$ & & $12,100(\geq 5,000)$ & $51(\geq 50)$ \\
\hline Lt. Ulnar & $1.7(\leq 4.2)$ & & $9,600(\geq 5,000)$ & $51(\geq 50)$ \\
\hline Lt. Radial & $1.3(\leq 2.0)$ & & $5,000(\geq 5,000)$ & $54(\geq 50)$ \\
\hline Rt. Peroneal & $3.2(\leq 6.0)$ & & $8,900(\geq 2,500)$ & $41(\geq 40)$ \\
\hline Rt. Tibial & $3.3(\leq 6.0)$ & & $6,200(\geq 5,000)$ & $40(\geq 40)$ \\
\hline Lt. Peroneal & $3.5(\leq 6.0)$ & & $8,500(\geq 2,500)$ & $43(\geq 40)$ \\
\hline Lt. Tibial & $2.6(\leq 6.0)$ & & $6,500(\geq 5,000)$ & $43(\geq 40)$ \\
\hline \multicolumn{5}{|l|}{ Sensory nerves } \\
\hline Rt. Median & $2.1(\leq 3.6)$ & 3.0 & $10(\geq 10)$ & $61(\geq 45)$ \\
\hline Rt. Ulnar & $1.9(\leq 3.4)$ & 2.8 & $12(\geq 10)$ & $56(\geq 45)$ \\
\hline Rt. Radial & $1.6(\leq 2.0)$ & 2.3 & $11(\geq 10)$ & $64(\geq 40)$ \\
\hline Lt. Median & $2.3(\leq 3.6)$ & 3.1 & $12(\geq 10)$ & $59(\geq 45)$ \\
\hline Lt. Ulnar & $1.9(\leq 3.4)$ & 3.4 & $11(\geq 10)$ & $57(\geq 45)$ \\
\hline Lt. Radial & $2.0(\leq 2.0)$ & 2.7 & $14(\geq 10)$ & $66(\geq 40)$ \\
\hline Rt. Sural & $2.7(\leq 3.5)$ & 3.8 & $7(\geq 10)$ & $42(\geq 40)$ \\
\hline Lt. Sural & $2.9(\leq 3.5)$ & 4.2 & $7(\geq 10)$ & $42(\geq 40)$ \\
\hline Rt. Superficial peroneal & $2.5(\leq 3.5)$ & 3.7 & $11(\geq 10)$ & $43(\geq 40)$ \\
\hline Lt. Superficial peroneal & $2.4(\leq 3.5)$ & 3.9 & $12(\geq 10)$ & $43(\geq 40)$ \\
\hline
\end{tabular}

The numeric values in parentheses refer to normal laboratory values. Rt., right; Lt., left.

cally abnormal intensity in the deep cerebella nuclei in the T2weighted image or DWI. These cases all demonstrated changes within the deep cerebella nuclei. Therefore this area is likely to be the most sensitive to the effects of metronidazole toxicity and also the most observable manifestation of metronidazole toxicity. In addition, neither case had demonstrated evidence of significant mass effect. However, only our case showed bilateral symmetric hyperintense lesions in the superior olivary nuclei of the dorsal pons on the MR imaging of the brain. In each of the cases, including ours, the symptoms rapidly improved following cessation of metronidazole therapy.

MIE may produce symptoms of progressive gait disturbance, ataxia and dysarthria, and toxic peripheral neuropathy does symptoms of sensory ataxia, tingling sensation and paresthesia. Unfortunately, our patient showed only dysarthria and a tingling sensation of hands because of her paraplegia due to her spinal cord injury. This made it very difficult to diagnosis for her symptoms. Another obstacle was that paraplegia left her with dull sensation to her lower extremities, which meant that the tingling would not have been noticed there correctly. A tingling sensation is early symptom of PPN. However, we didn't doubt that symptom caused by peripheral polyneuro pathy, because that was uncommon in patient with spinal cord injury. And, because we could not examine ataxia and gait disturbance, we did not know MIE. Also, NCS study and brain MRI were not preceded on early stage. As a result we could not check for PPN earlier.

MIE is a reversible disease which can improve a few weeks after discontinuation of metronidazole ${ }^{1,5,6}$. Therefore, correct and early diagnosis is very important to treat patients with MIE. But considering the rare situation the patient was in, MIE or other neurological problems may be difficult to diagnose, especially when such side effects are not common. The importance of an active differential diagnosis can be seen in our case. Whether the initial tingling of the hands may have been a sign of early MIE or other neurological problem, it would be the best to consider all possible causes before the onset of new symptoms, especially when the severity of the disease is measured by the time of detection.

\section{CONCLUSION}

MIE and toxic neuropathy can be occurred by neurologic complications of metronidazole. MIE and toxic neuropathy can produce many kinds of neurologic symptoms. In special circumstances such as a paraplegia patient, these symptoms may not be present. However, if a tingling sensation in the hands or one of cerebellar symptoms such as dysarthria, dysmetria, and so on, is observed in patient with spinal cord injury, it is important to consider and to evaluate a possibility of peripheral polyneuropathy, or encephalopathy especially, 
patient take some metronidazole, because an early detection means a better recovery.

\section{REFERENCES}

1. Ahmed A, Loes DJ, Bressler EL: Reversible magnetic resonance imaging findings in metronidazole-induced encephalopathy. Neurology 45:588-589, 1995

2. Bahn YC, Kim EY, Park CG, Park HC: Metronidazole Induced Encephalopathy in a Patient with Brain Abcess. J Korean Neurosurg Soc 48:301-304, 2010

3. Finegold SM: Metronidazole. Ann Intern Med 93:585-587,
1980

4. Heaney CJ, Campeau NG, Lindell EP: MR imaging and Diffusion-Weighted Imaging Changes in Metronidazole (Flagyl)Induced Cerebellar Toxicity. Amedican J Neuroradiol 24:16151617, 2003

5. Horlen CK, Seifert CF, Malouf CS: Toxic metronidazole- induced MRI changes. Ann Pharmacother 34:1273-1275, 2000.

6. Kim KH, Choi JW, Lee JY: Two cases of metronidazole-induced encephalopathy. Korean J Gastroenterol 45:195-2000, 2005

7. Kusumi RK, Plouffe JF, Wyat RH, Fass RJ: Central nervous system toxicity associated with metronidazole therapy. Ann Intern Med 93:59-60, 1980 